\title{
MOST CANCERS ARE CAUSED BY BAD LUCK MUTATIONS: SCIENTIFIC EXPLANATION?
}

Cancer incidence is increasing worldwide and about $50 \%$ of new cases are diagnosed in Asian continent alone as per IARC 2018. Asia Pacific region in particular carries the highest burden in the world which is understandable as some of the most populous countries in the world such as China, India, Pakistan, Bangladesh, Indonesia and Japan are part of this region. Robust data from across the globe correlating 'Environmental and Lifestyle Factors' with various types of cancers are non-refutable. For instance, 2017 data of 'Karachi Cancer Registry' as well as 'Punjab Cancer Registry' show 'Oral Cancer' as the most common cancer in males and the second most common in females of Karachi and Punjab. As per our records at Aga Khan University Hospital, over 95\% of oral cancer patients are addicted to chewing or smoking. Similarly, there is correlation between certain types of lung cancers with smoking and liver cancers with chronic hepatitis. However, there are still a large number of cancers which arise out of the blue with no known risk factors. A recent paper puts random fatal mutations arising in otherwise healthy cells as the leading cause of 'Cancer' with the 'Environment' second and the inherited mutations a distant third. ${ }^{[1]}$ Those cancers without any known or well-established risk factors include some of the most lethal cancers such as 'Brain gliomas,' 'Pancreatic cancer,' 'Ovarian cancers' and many others including even 'Breast Cancer.'

Question is then asked, particularly by the patient; "why am I suffering from this cancer in spite of healthy habits and no obvious risk factors?' In a study from John Hopkins, which created much controversy, it was concluded that random mutations in healthy cells may explain two thirds of all cancers. Through an ingenious mathematical model, it was calculated that as the number of stem cell divisions in a tissue rises so does the chances of cancer affecting that site. For instance in colorectal where total stem cell divisions are much higher than duodenum, there is a much higher lifetime risk of developing colorectal cancer than duodenal cancer ${ }^{[1]}$

Correspondence: Shahid Pervez,

Department of Pathology and Laboratory Medicine, Aga Khan

University Hospital, Karachi, Pakistan.

Email: shahid.pervez@aku.edu keeping in mind that most photocopying errors happen at the time of cell divisions when a cell doubles its DNA before equal distribution of genetic material in two daughter cells. Tomasetti et al. ${ }^{[1]}$ calculated the relative impact of environment, inheritance and random DNA mutations as causative factors in cancer initiation, from a UK cancer database. Interestingly, they showed that the impact of these factors varies from one cancer type to another. In case of prostate, bone and brain cancers, $95 \%$ tumours were due to random errors in DNA mutations, whereas in lung cancer, $65 \%$ of cases were caused by environmental factors related mutations and $35 \%$ were due to DNA copying errors.

To explain this further, we need to go back to basics i.e., DNA. DNA is a fragile molecule that is continually under assault not only from agents in the environment and our diet but also even from normal processes that take place within the cell. ${ }^{[2,3]}$ It is, therefore, critically important that damage to our genetic material is recognised and efficiently removed. As per estimate, an adult human body contains somewhat around 125 trillion cells. It has been calculated that in a single day, DNA in one cell suffers about 20,000 single-strand breaks and 10-20 doublestrand breaks. Single-strand breaks are easy to repair as complimentary sequences are present; however, doublestrand breaks are difficult to repair as both strands of the double-helix are affected such that there is no immediately available template in the form of an intact complementary strand to use for correcting the lesion. It also involves the risk of losing a whole part of chromosome and potentially hundreds and thousands of genes. ${ }^{[2-4]}$ Most errors happen at the time of cell replication. Keeping in mind trillions of cells in human body, the propensity of DNA damage and DNA repair genes such as BRCA1 and BRCA2 $2^{[4,5]}$ which also are prone to develop mutations, one actually ponders instead that 'Why cancer is so uncommon?.'

This aspect further highlights the need for screening programs as the only way to diagnose and possibly cure non-preventable cancers is to diagnose at a stage when they are localised and curable. This is particularly relevant to cancers such as breast, colorectal and prostate cancers where mammogram, colonoscopy and simple 
prostate-specific antigen testing may make a difference between life and death.

Finally, for the common person, it is difficult to comprehend that 'Cancer' is not 'one disease' and that there are several hundred types of cancers, each one is unique in terms of its biology and in particular its management. As cancer registries lump all types of cancers in a particular organ, it is likely to be conceived that all patients with a cancer of a particular organ are likely to meet the same fate irrespective of its subtypes which is not true. For instance, most common germ cell tumour of the testis 'Seminoma' even if extensively metastasised, shows a cure rate of about 95\% with current treatment modalities while a brain tumour even if localised, by virtue of its location alone is likely to be associated with much higher mortality.

In a nutshell, 'Cancer' is an intriguing disease in which both genetic and epigenetic (environmental) factors play an important role and the fact that many cancers are nonpreventable, early detection is the only effective strategy which may make a difference not only for an individual but also for the entire humanity at large.

Shahid Pervez

Department of Pathology and Laboratory Medicine, Aga Khan University Hospital, Karachi, Pakistan Received: 08 March 2019 / Accepted: 18 March 2019

\section{References}

1. Tomasetti C, Li LU, Vogelstein B. Stem cell divisions, somatic mutations, cancer etiology, and cancer prevention. Science 2017;355:1330-4.

2. Lindahl T. The intrinsic fragility of DNA (Nobel lecture). Angew Chem Int Ed Engl 2016;55:8528-34.

3. Lindahl T. Instability and decay of primary structure of DNA. Nature 1993;362:709-15.

4. Lindahl T, Modrich P, Sancar A. The 2015 nobel prize in chemistry. The discovery of essential mechanisms that repair DNA damage. J Assoc Genet Technol 2016;42:37-41.

5. Shahid T, Soroka J, Kong E, et al. Structure and mechanism of action of the BRCA2 breast cancer tumor suppressor. Nat Struct Mol Biol 2014;21:962-8. 